This item was submitted to Loughborough's Research Repository by the author.

Items in Figshare are protected by copyright, with all rights reserved, unless otherwise indicated.

\title{
Multilayer plasma patterns in paralleled and coupled atmospheric glow discharges
}

PLEASE CITE THE PUBLISHED VERSION

PUBLISHER

(c) IEEE

VERSION

VoR (Version of Record)

\section{LICENCE}

CC BY-NC-ND 4.0

\section{REPOSITORY RECORD}

Yang, Y., J.J. Shi, J.E. Harry, John W. Proctor, Colin P. Garner, and Michael G. Kong. 2019. "Multilayer Plasma Patterns in Paralleled and Coupled Atmospheric Glow Discharges". figshare.

https://hdl.handle.net/2134/5225. 
This item was submitted to Loughborough's Institutional Repository (https://dspace.lboro.ac.uk/) by the author and is made available under the following Creative Commons Licence conditions.

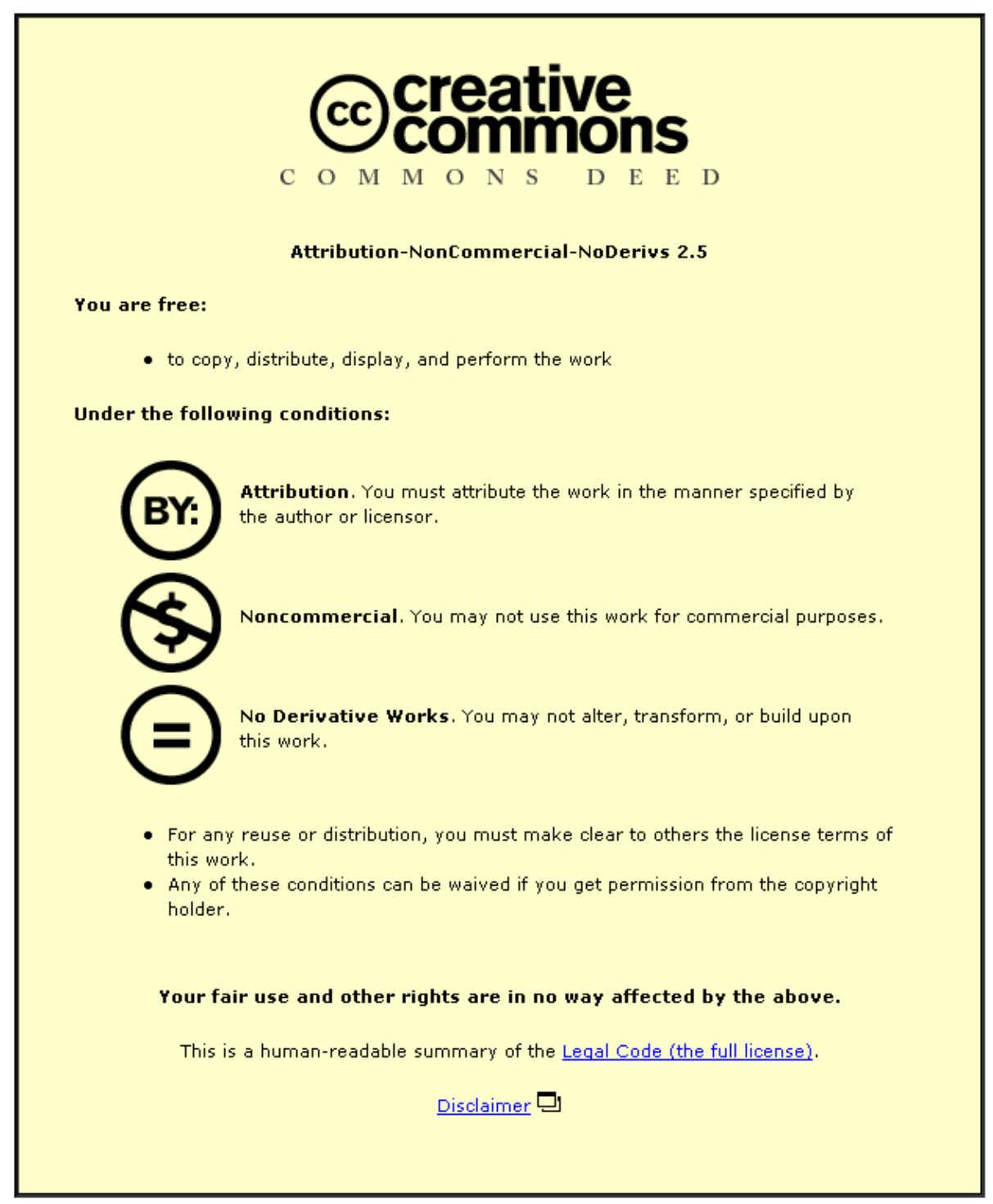

For the full text of this licence, please go to: http://creativecommons.org/licenses/by-nc-nd/2.5/ 


\title{
Multilayer Plasma Patterns in Paralleled and Coupled Atmospheric Glow Discharges
}

\author{
Y. Yang, J. J. Shi, J. E. Harry, J. Proctor, C. P. Garner, and M. G. Kong
}

\begin{abstract}
We report observations of multilayer plasma patterns in multiple atmospheric glow discharges sustained simultaneously with a single power supply. Depending on operation conditions, these atmospheric glow plasmas either operate in parallel, seemingly independent of one another, or undergo structural coupling. In both scenarios, multilayer structures are observed. These selforganized plasma patterns are stable and their presence remains even when individual atmospheric glow plasmas couple with one another.
\end{abstract}

Index Terms-Atmospheric pressure glow discharges, plasma coupling, self organization, striation.

ELF-ORGANIZATION is a natural process of sponta$\checkmark$ neous formation of ordered structures or patterns usually under unfavorable external conditions. Self-organized patterns are found in a variety of nonlinear and nonequilibrium systems, for example the Turing patterns observed in nonequilibrium chemical reactions [1] and bifurcation patterns formed in rapid growth of bacterial colonies [2]. Typically a self-organized pattern consists of seemingly identical parts or components that are aligned with respect to one another in an ordered fashion. The spontaneous alignment of the individual components is often a result of a globally self-adjusted adaptive response to extreme external conditions.

In the context of gas discharge plasmas, including space plasmas and low-pressure glow discharges, self-organization of complex plasma patterns has commanded much attention. Broadly there are two different groups of self-organized patterns, namely: 1) self-organization of multiple plasmas simultaneously sustained between two parallel electrodes [3], [4], and 2) self-organized multilayer patterns developed along the length of a single plasma column [5], [6]. In this paper, we report observation of multilayer structures in each of several self-organized atmospheric pressure glow discharges (APGD) sustained simultaneously with a single power source. Therefore, the observed self-organization is an amalgamation of the above-mentioned two groups of self-organized plasma patterns. To our knowledge, this is the first observation of interacting self-organized plasma systems in atmospheric pressure glow discharges.

Manuscript received June 25, 2004; revised November 18, 2004.

Y. Yang, J. Proctor, and C. P. Garner are with the School of Mechanical and Manufacturing Engineering, Loughborough University, Leicestershire LE11 3TU, U.K.

J. J. Shi, J. E. Harry, and M. G. Kong are with the Department of Electronic and Electrical Engineering, Loughborough University, Leicestershire LE11 3TU, U.K. (e-mail: m.g.kong@lboro.ac.uk).

Digital Object Identifier 10.1109/TPS.2005.845099

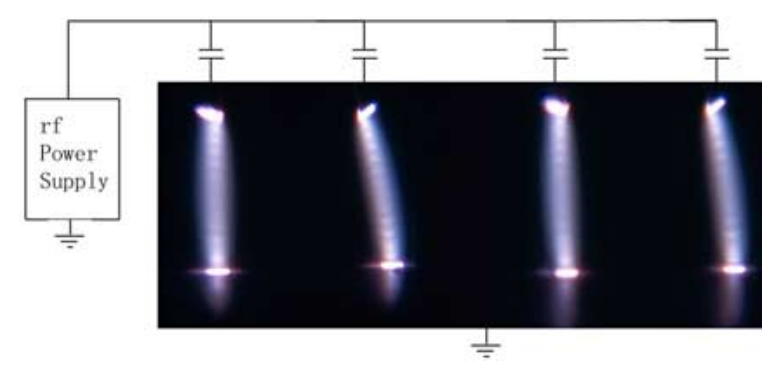

Fig. 1. Four parallel atmospheric glow plasmas are sustained simultaneously by a single power supply via capacitors. Multilayer structure is clearly seen in each atmospheric glow discharge.

Our plasma rig has a ground stainless-steel plate electrode and several parallel copper pin electrodes which are connected together to a common power source. The power supply provides sinusoidal high voltages at a frequency between 10 and $100 \mathrm{kHz}$. A capacitor of $22 \mathrm{pF}$ is added in series with each pin electrode so as to stabilize the generated plasmas. Helium flow of nominally $5 \mathrm{slm}$ is fed through the gap between the plate electrode and the pin electrodes. Electrode gap is nominally $8 \mathrm{~mm}$ and the pin electrodes have a diameter of $0.8 \mathrm{~mm}$. Electrical measurements were made, via voltage and current probes, with a digital oscilloscope (500 MHz and $2 \mathrm{GS} / \mathrm{s}$ ). The rise time of the current probe is 2 ns and so it should pick up nanosecond filamentary discharges. In general, the current traces were smooth and free of any nanosecond spikes. These observations suggest that the generated atmospheric plasmas are glow discharges.

Fig. 1 shows the photographs of a multilayer pattern formed in each of four atmospheric pressure glow plasmas sustained simultaneously between four powered pin electrodes and a common plate electrode. The separation distance between two adjacent pin electrodes is at least $5 \mathrm{~mm}$. Similar to that in comparable experiments, the four stabilizing capacitors are important to achieve simultaneous generation of multiple atmospheric plasmas [7], [8]. In each of the four atmospheric plasmas, there are four bright layers near each electrode. It is worth mentioning that the four bright layers near the bottom plate electrode seem very similar to that near the pin electrode. This suggests that surface conditions of the two electrodes are not a dominant factor in striation formation. Given that a pin electrode may facilitate electric field enhancement near its tip and the plate electrode does not, the symmetric multilayer structure of Fig. 1 highlights a distinct nature of self-organization. The multilayer plasma pattern is sometimes known as striation structure [5], [6]. In our experiments, the four striated atmospheric glow discharges were very stable and appeared stationary even though their excitation was at a higher frequency. 


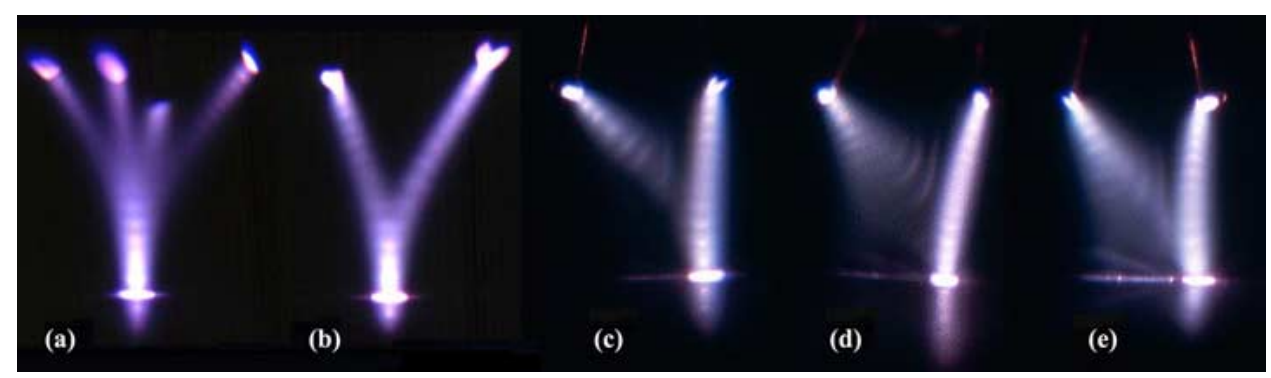

Fig. 2. Striated atmospheric glow plasmas undergo various forms of coupling with symmetrical coupling in (a) and (b) and asymmetric coupling in (c), (d), and (e).

When the distance between two adjacent pin electrode is less than $5 \mathrm{~mm}$, individual atmospheric glow plasmas start to couple to one another. In Fig. 2(a), four atmospheric glow discharges leave their respective pin electrodes and join together in a common cylindrical column before reaching a common root on the plate electrode. Multilayer patterns are seen near each of the four pin electrodes as well as in the common cylindrical column linking the plate electrode. However, the multilayer structure in the common cylindrical column appears much brighter. Fig. 2(b) shows the coupling between two atmospheric glow discharges, exhibiting similar features. Multilayer structures are clear near the two pin electrodes and they develop spatially onto their common root via a cylindrical column. The multilayer structure along this cylindrical column is more intense than those near the pin electrodes. In general, individual atmospheric glow plasmas are coupled together in a spatially symmetric manner. They are stable and seemingly stationary.

It is possible for the coupling among individual glow discharges to be asymmetric, although current and voltage measurements do not appear to differentiate the operation conditions for symmetric coupling of Fig. 2(a) and (b) from that for asymmetric coupling. The two different symmetric appearance is, however, not random, and may be influenced by surface conditions of the electrodes. In Fig. 2(c)-(e), the coupling between two individual atmospheric glow plasmas are no longer symmetric. One atmospheric glow discharge is established between its pin electrode and the plate electrode, whereas the other is formed between its own pin electrode and a point along the length of the first plasma. For convenience of discussion, the first is referred to as the primary plasma and the other as the secondary plasma. The primary plasma is similar to that observed in Fig. 2(a) in that multilayer structures are found near both electrodes and that the two multilayer structures are similar in their visual appearance. The secondary plasma, however, has a fan-shaped root region joining the primary plasma, and the region near the fan-shaped root is striated. As the applied voltage increases, the fan-shaped root region begins to spread along the length of the primary glow plasma as seen in Fig. 2(d). With further increase in the applied voltage, this spreading extends to the plate electrode shown in Fig. 2(e). In all cases of asymmetric coupling, individual glow discharges are stable.

In conclusion, self-organized plasma patterns formed in an array of multiple glow discharges at atmospheric pressure have been successfully sustained by one common high frequency power source. With a separation distance larger than $5 \mathrm{~mm}$ and an appropriate applied voltage, each of these individual glow discharges develops a seemingly identical multilayer pattern near their electrodes. When their separation distance is reduced to below $5 \mathrm{~mm}$, individual glow plasmas undergo either symmetric or asymmetric couplings. Through a self-organization, their roots at the earthed plate electrode merge together to form a common cylindrical region such that the coupled plasma cluster takes the form of a tree-like configuration. Multilayer patterns remain along the length of the coupled plasma cluster. Asymmetric coupling is also observed in a two-plasma system with one glow discharge formed between its electrodes and the other formed between its pin electrode and a root point along the length of the first glow discharge. With increasing applied voltage, the root point evolves into a fan-shaped region that can spread onto the earthed plate electrode. In all cases, striation patterns are clearly seen and appear stationary. At this stage, it remains unclear which underlying mechanisms are responsible for the spontaneous formation of multilayer structures in multiple simultaneously sustained plasmas. While ion waves have been considered as the mechanism for the formation of double layers in space plasmas and could also be responsible for the observed striation reported here, much needs to be done before this can be substantiated.

\section{REFERENCES}

[1] Q. Quyang and H. L. Swinney, "Transition From a Uniform State to Hexagonal and Striped Turing Patterns," Nature, vol. 352, pp. 610-612, Aug. 1991

[2] J. Y. Wakano, S. Maenosono, A. Komoto, N. Eiha, and Y. Yamaguchi, "Self-organized pattern formation of a bacteria colony modeled by a reaction diffusion system and nucleation theory," Phys. Rev. Lett., vol. 90, Jun. 2003.

[3] I. Muller, C. Punset, E. Ammelt, H.-G. Purwins, and J. P. Boeuf, "Selforganized filaments in dielectric barrier glow discharges," IEEE Trans. Plasma Sci., vol. 27, no. 1, pp. 20-21, Feb. 1999.

[4] W. Breazeal, K. M. Flynn, and E. G. Gwinn, "Static and dynamic twodimensional patterns in self-extinguishing discharge avalanches," Phys. Rev. E, Stat. Phys. Plasmas Fluids Relat., vol. 52, pp. 1503-1515, Aug. 1995.

[5] Y. Yang, J. Shi, J. E. Harry, J. Proctor, C. P. Garner, and M. G. Kong, "Multilayer Plasma Patterns in Atmospheric Pressure Glow Discharges," IEEE Trans. Plasma Sci., vol. 33, pp. 298-299, Apr., 2005.

[6] D. Diebold, C. E. Forest, N. Hershkowitz, M.-K. Hsieh, T. Intrator, D. Kaufman, G.-H. Kim, S.-G. Lee, and J. Menard, "Double-layer-relevant laboratory results," IEEE Trans. Plasma Sci., vol. 20, no. 12, pp. 601-606, Dec. 1992.

[7] H. Mase, T. Fujiwara, and N. Sato, "Capacity-coupled multidischarge for atmospheric plasma production," Appl. Phys. Lett., vol. 83, pp. 5392-5394, Dec. 2003.

[8] J. E. Harry and A. Yahya, "Conditions for coalescence of multiple electric discharges," in Proc. Inst. Electr. Eng. A, vol. 139, Jul. 1992, pp. 210-212. 